\title{
Trending Technologies for Indoor FM: Looking for "Geo" in Information
}

\author{
M. Gunduz ${ }^{\mathrm{a}}$, U. Isikdag ${ }^{\mathrm{b} *}$ M. Basaraner ${ }^{\mathrm{a}}$ \\ a Yildiz Technical University, Dept. of. Geomatic Engineering, Istanbul Turkey - memogedo@gmail.com; mbasaran@yildiz.edu.tr \\ ${ }^{\mathrm{b}}$ Mimar Sinan Fine Arts University, Dept. of Informatics, Istanbul Turkey - uisikdag@ gmail.com
}

KEY WORDS: Facility Management, GIS, BIM, Geoinformation, Text Mining

\begin{abstract}
:
Today technological developments in the Architecture Engineering and Construction (AEC) industry provides opportunities to build huge and complex buildings and facilities. In order to operate these facilities and to meet the requirements of the occupants and also to manage energy, waste and to keep all facility services operational, several Facility Management (FM) solutions were developed. This paper starts by presenting a state of art review of research related to Indoor Facility Management Systems. Later, a textual analysis focused to identify the research trends in this field is presented in the paper. The result of the literature review and textual analysis indicates that current research in Indoor FM Systems is underestimating the role of Geoinformation, Geoinformation models and systems.
\end{abstract}

\section{INTRODUCTION}

Facilities Management is known as a set of multi-disciplinary activities to manage the operations of a building or a facility following the construction phase of a building's life cycle. This phase is known as the "Operation" or "Facilities Management" phase of the building in the AEC industry. The Facilities Management professionals deal with many complex systems that operate synchronously to manage the quality of services in the building such as water, air, gas, electricity. Furthermore, the automation of building services and the security of the building is also the focus of this domain. In addition, the Facilities Management also deals with establishing and maintaining the comfort of the occupants (i.e. the indoor comfort). Due to this nature of the Facilities Management, FM professionals are required to work with many different systems. This nature of the domain also brings the requirement of working with different software or information systems, some of which are integrated and some of which are not. A single system is not sufficient to satisfy the needs of a facility complex. Thus, one of the earlier research topics related to Facilities Management technologies and FM Information Systems was the integration at both at information model and information system levels. Model Based Integration which is facilitated by Building Information Models(BIMs) is still an important research topic in the domain. Furthermore, in parallel with the advancements in visualization and immersion technologies the application of Virtual and Augmented Reality technologies to the FM domain is also becoming popular.

In order to, i.) understand and interpret the influence of different technologies and ii.) explore the role of Geoinformation, Geoinformation systems and Geoinformation technologies this research has implemented 2 different methods. First method implemented was a comprehensive literature search and review which has been made based on papers reached by using "Facilites Management", "Building Information Modeling", "GeoInformation Systems" , "GIS", "Augmented Reality", "Indoor", "Indoor Positioning" keywords. Academic Search Engines used in the literature search was Elsevier Sciencedirect, Google Scholar, and American Society of Civil Engineering Library Database.

The findings of the literature search are presented in this paper as grouped under 6 categories which are Virtual \& Augmented reality, Routing \& Navigation Methods, Energy Management Focused Technologies, Positioning and Sensors, BIM based solutions, Geoinformation based solutions, and Integration Efforts (see. Section 2).

The second method implemented in the research was textual analysis. Text mining techniques were used as the textual analysis approach (see. Section3). The approach is utilized to determine the general research trends based on the papers that were found at the literature search phase. Text mining refers a series of computer-aided statistical techniques with the aim of deriving meaningful information from structured or unstructured texts. As a result of the text mining analysis, words most commonly occur in the literature were first determined and then, visualized in form of word clouds. Finally, a hierarchical clustering of words is generated and this clustering output is visualized in form of a dendrogram. The analysis of the dendrogram is presented in the conclusion section.

\section{TRENDING TECHNOLOGIES}

This section provides an overview of trending technologies related to Indoor Facilities Management. The findings of the literature search and review process are presented here in 6 categories.

\subsection{Virtual \& Augmented Reality}

The first category of technologies that were notified was Virtual and Augmented Reality. These technologies were used to support communication between stakeholders, which plays a crucial role for facility management. For example, Shi et al. (2016) proposed a BIM based online communication system which uses VR in order to make the interactions more efficient. The system makes use of a BIM by implementing it in a game engine called Unity. With the help of Oculus Rift Head Mounted Device, stakeholders can share and experience same virtual facility space even if they are not at the same location.

\footnotetext{
Corresponding author
} 
As another example, Lee and Akin (2011) who developed an Augmented Reality-Based Operation and Maintenance Fieldwork Facilitator (AROMA-FF). According to researchers, this application saved $51 \%$ of time spent at a FM task. System also gathers performance data from sensors in real-time that saved $8 \%$ of time at field tasks.

According to a survey of information technologies used in facility management (FM), conducted by Gheisari et al. (2014), FM professionals generally $(92 \%)$ use electronic format files such as Pdf, word to exchange information and the percentage of use of 2D/3D CAD/BIM is 39\%. Their knowledge level of BIM was also found $60 \%$, which indicates that FM Professionals are aware of BIMs. It is reported that Facility Managers are keen to utilize BIM \& Augmented Reality solutions to complete FM operations with minimum effort.

The Augmented Reality systems require visual markers that need to be set in the facilities for navigation and maintenance purposes. Installing these markers could not be esthetical and time consuming. Koch et al. (2014) presented a framework that can use natural markers such as exit signs, fire signs etc. for facilitating FM tasks. Ammari and Hammad (2014) combined BIM, video, sensors and Real Time Location Systems (RTLS) at a BIM-Based Markerless Mixed Reality (BIM3R) framework. System can recognize features from the visual data and compare component's deformation via edge detection feature.

\subsection{Energy Management Focused Technologies}

As stated by Becerik-Gerber et al. (2012), "for controlling and monitoring energy, FM personnel rely on energy management systems, which measure the energy usage at a building or zone level over a period of time and sometimes in real-time." Energy efficiency is considered as a vital topic for Facilities Management. As indicated by Cao et al. (2015), buildings consume $36 \%$ of total energy of a city. Accurate decisions may help to reduce the energy waste. But, it is a hard job for the Facility Managers to cope manually with scheduling tasks, fulfill occupant requests, and solve maintenance issues. At this point, an artificial intelligence method, Case-Based Reasoning (CBR) is proposed by the authors. The proposed system consisted of three main components; input, data analysis and output. The system learns and clusters the system requests in time and helps the Facility Manager for the decision of work priority in an energy efficient manner.

\subsection{Positioning and Sensors}

Indoor positioning plays a key role for Asset and Facility management systems, but lack of stability and high accuracy are the restrictions of indoor positioning systems for producing efficient outcomes for FM domain. In fact, the research in the field focuses on developing more accurate systems. Li et al. (2016) classified 7 most frequent real-time positioning systems as Radio Frequency Identification (RFID), Global Positioning System (GPS), Ultra-wideband (UWB), Vision Analysis, Wireless Local Area Network (WLAN), Ultrasound, and Infrared (IR). For example, a system developed by Song and Wang (2011) was named as Space Annotator. Space Annotator used Time of Arrival (TOA) based ultrasound system for submeter precision and able to perform $\mathrm{x}-\mathrm{y}-\mathrm{z}$ (3D) positioning. Nevertheless, as a consequence of the poor penetration capacity and limited scope, ultrasound method results may fail at incompatible environments.

On the other hand, there are relatively new positioning technologies such as Bluetooth, infrared, audible sound, IndoorGPS, Inertial Measurement Units (IMU), and magnetic signals which can be used to aid FM operations and tasks. According to Akula et al. (2011), infrastructure-based positioning systems provide better results than infrastructure independent tracking systems such as Personal Dead Reckoning (PDR) or Personal Odometry System (POS). On the other hand, obviously there could be situations that pre-installed infrastructures are non-operative. Therefore, researchers proposed an Integrated Tracking System (ITS), which uses both infrastructure-based and independent system together. As a result, it is possible to get seamlessly and more accurate indoor positioning results.

Obviously, it is impossible to measure or track every parameter that affects occupants' comfort in facilities. For this reason, attributes of Indoor Environmental Quality (IEQ) should be determined. Lai and Yik (2009), investigated some IEQ factors in the residents' perspective of importance. As a result, thermal comfort regarded as the most important IEQ parameter. Thermal condition is important for health and comfort of passengers and for efficient energy consumption. A BIM-WSN (Wireless Sensor Network) system presented by Marzouk and Abdelaty (2014) monitored thermal condition for this purpose. System acquired thermal data via sensors and those nodes were connected to BIM database.

\subsection{BIM Based solutions}

BIM based solutions and technologies are the most commonly focused research fields that is related to the Indoor FM. Terreno et al. (2015) presented a survey among key FM personnel, who highlighted benefits of BIM integration with FM as; better collaboration, more detailed strategic planning, easier data retrieval, more proactive maintenance, and increased employee productivity.

At different phases of a building's life cycle, same information for a location may have been collected repeatedly. The utilization of BIM processes may prevent this wasted effort but as the Liu and Issa (2013) reported that there is a lack of BIM knowledge between the FM stakeholders. The authors conducted a survey with the intention of exploring issues in BIM for Facility Management and responses of questions showed that, information related to the "name of the manufacturer", "location of the equipment", and "maintenance repair instruction" needed in FM work orders. In fact, most of respondents stated that they don't have such a model with related FM information.

Condition assessment is a necessary process of Facilities Management to keep systems and components functional. Kim and $\mathrm{Yu}$ (2016) defined a method for BIM-based condition assessment comprised of planning, inspection, priority analysis and update stages. Therefore, FM managers can modify data directly from BIM-based model. Facility Management includes diverse use cases. Facility managers may need different data from FM data sources for different circumstances. Kang and Choi (2015) investigated a BIM perspective for definition of a metadata structure. That structure allows to define and expand user perspectives and makes it possible to connect heterogeneous facility management data.

As Mc Arthur (2015) describes, there are four main challenges for sustainable operations management in buildings. 1) Identification of critical information for operational decisions. 2) Implying real-time operation and monitoring inputs to BIM. 3) Ease to modify/create BIM. 4) Incomplete building documentation and related uncertainty. The author offered a framework to overcome these challenges.

Management of more complex facilities (such as hospitals) brings more complex FM tasks. For example, benefits of BIM 
usage at Healthcare facility management is studied by Wang et al. (2016).

BIM usage at FM operations may save the time which is spent by FM personnel on spotting the place of an issue, getting the related documents, matching the documents and location and updating work order to FM system. Visualizing a problem and inspecting the cause of it via fault trees was made possible with the research of Motamedi et al. (2014). Their system was called as FM-VAS (FM Visual Analytics System). The system provides visual representation of the related FM issues (Figure 1 ) by integration of data from various sources (e.g. Construction-Operation Building Information Exchange (COBie) documents, BIM, and Computerized Maintenance and Management System (CMMS)).

An effort for visualizing Facility Management helped in making spatial trend analysis and to determine spatial relationships between FM activities.

Some facilities like airports or governmental structures may need access control for security or authorization needs. Skandhakumar et al. (2016) proposed a graph representation of BIM that can be used for routing/pathfinding or accessibility related functions.

Motamedi et al. (2011) developed a BIM based distributed data storage system based on RFID tags. The research of Motawa and Almarshad (2013) aimed to provide a BIM based system that not only captures and stores the facility information but also offers support for preventive or corrective decisions in the future events. Wetzel and Thabet (2015) were focused on the safety side. They presented a BIM-based framework to reduce the risks of injury and provide better personnel security. Their framework provided better information access for maintenance and repair staff.

An analysis was presented by Khaja et al. (2016) on software that transfers information between BIM and facility management systems, and it has been observed that this transfer reduces the cost and time in FM processes.

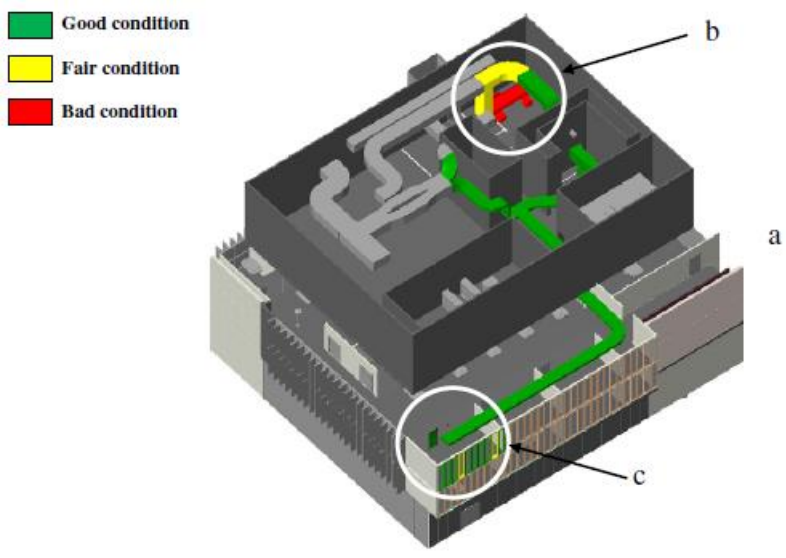

Figure 1. Visualization of FM related issues. (adopted from Motamedi et al. , 2014).

\subsection{Geoinformation based solutions}

Evacuation management is one of the primary domains which makes use of Geoinformation for indoor routing and navigation for Facilities Management. For instance, Atila et al. (2014) proposed a solution (data and network generation, visualization and evacuation model) for fire evacuation scenarios. In a disaster scenario, routing for shortest exit path in order to evacuation may not be sufficient. People may rush to a common single exit and a confluence might happen. Similarly, research of Choi and Lee (2009) aimed to calculate temporal changes during evacuation with proposed agent-based model.

Hua et al. (2014) presented a post occupancy evaluation (POE) study, which provided crucial data for FM purposes. With the aim of understanding of occupant satisfaction related to the indoor environment quality, factors such as air movement, $\mathrm{CO} 2$ level, light level, temperature were investigated by using a Geoinformation System during the study.

\subsection{Integration Efforts}

It is foreseeable that the integration of BIM and Geoinformation would be very beneficial for FM domain. The literature review revealed several studies with such focus. Information interoperability is also important for reusability and extensibility. For instance, Kang and Hong (2015) proposed a software architecture which integrates BIM and Geoinformation models with extract, transform and load (ETL) methods. For example, Wu et al. (2014) offered a GIS-BIM based Virtual Facility Energy Assessment (VFEA) framework. Liu and Issa (2012) have completed an investigation for testing a manual BIM and GIS integration by making use of off-the-shelf software. A campus building used for testing the interoperability of ArcGIS, Revit and AutoCAD Civil 3D at system level. Integration of the systems is a difficult task when there are many phases of the facility lifecycle and generally needs of each phase are different. At the end, the information that is obtained at former phases may be required for the next phases. Therefore, phase specific systems, data sources and applications should be able to communicate. Shen et al. (2012) presented an agent-based, service-oriented approach in this manner.

\section{TEXT MINING ON THE LITERATURE RESOURCES}

Following the classification of the research subjects in 6 categories, the next stage of the research involved a textual analysis and mining process.

In this process first, all papers (that were investigated during the literature review stage) were converted into .txt format mainly from PDF files, using a PDFZilla software (one of the many packages that can be found to convert from PDF to TXT). In the following stage, the authors have implemented code in $\mathrm{R}$ language (by using R Studio environment) for text mining and analysis tasks. The development of the program code instead of using an off-the-shelf software package was due to author's intention of achieving flexibility and control at every stage of the process. The code implemented firstly executes the preprocessing tasks in the following order; 
a-Removing punctuation from the text

b-Removing numbers

c-Conversion of all text into lowercase

d-Removing of stop words. A standard set of stop words can be found at GitHub Arc12 (2016)

e-Removing of common word endings (i.e. -ing, -es)

f-Removing whitespace

It should be noted that the only filtering applied was filtering the standard stop words, and there was no other domain or context specific filtering applied. Following the preprocessing tasks (a-f), a document-term-matrix is constructed for achieving further analysis. A document-term matrix is a matrix that provides the frequencies of terms that occurs in a set of documents. In this type of matrix, the rows denote the documents while the columns represent the terms. In the next phase, frequencies of occurrence for each word is determined and provided as a table in R Studio. This occurrence frequency table is then manually copied by the authors to Tableau Public Software (Tableau,2016) for visualization purposes. The Tableau software is then used for generating an interactive bar chart and an interactive tree map. Figure 2 presents a snapshot of the interactive bar chart, the original form can be reached from

(https://public.tableau.com/views/IndoorFMTextMining01/Shee t1). Similarly Figure 3 presents a snapshot of interactive treemap while the original can be reached from (https://public.tableau.com/views/IndoorFMTextMining02/Shee t1). The readers are advised to check original interactive charts from the web, and try different visualizations changing the frequency value.

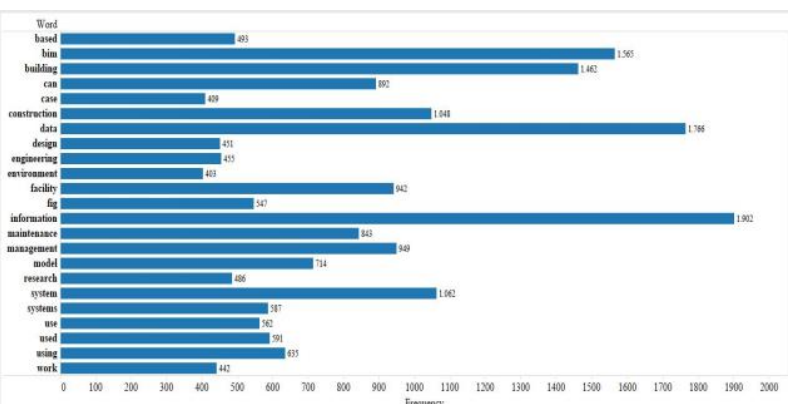

Figure 2: Frequency of occurrence for commonly occurring words (Bar chart View)

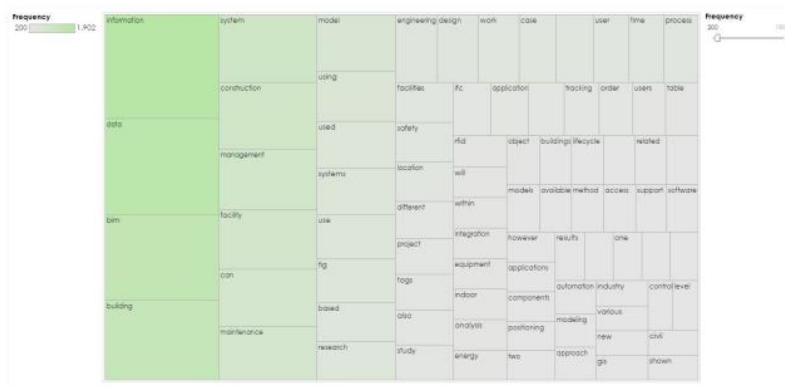

Figure 3: Frequency of occurrence for commonly occurring words (Tree map View)

When the minimum frequency count threshold is set to 600 empirically (as the value of 600 provided a clear set of domain related words in the effort where domain or context specific filtering not applied) the word list appeared as follows (in A-Z order):

$\begin{array}{ll}- & \text { bim } \\ \text { - } & \text { building } \\ \text { - } & \text { can } \\ \text { - } & \text { construction } \\ \text { - } & \text { data } \\ \text { - } & \text { facility } \\ \text { - } & \text { information } \\ \text { - } & \text { maintenance } \\ \text { - } & \text { management } \\ \text { - } & \text { model } \\ \text { - } & \text { system } \\ \text { - } & \text { using }\end{array}$

In order to re-group these words within a logical grouping, first the words "can", "using" are excluded as they are the "stop words" as well. The result of occurrence frequency analysis points out that "information" is the most frequent word, this is followed by "data" and then "bim". The frequency analysis showed that the research outputs related to Indoor FM systems, mainly focus on the value of information, along with modeling, representation and management of it. In the next stage of the text mining effort a word cloud is generated for visualization purposes, by using the $\mathrm{R}$ Studio as the coding environment and $\mathrm{R}$ code as the tool. In building up the word cloud, 100 words that most frequently occur in the text was selected. Figure 4 provides a representation of the word cloud.

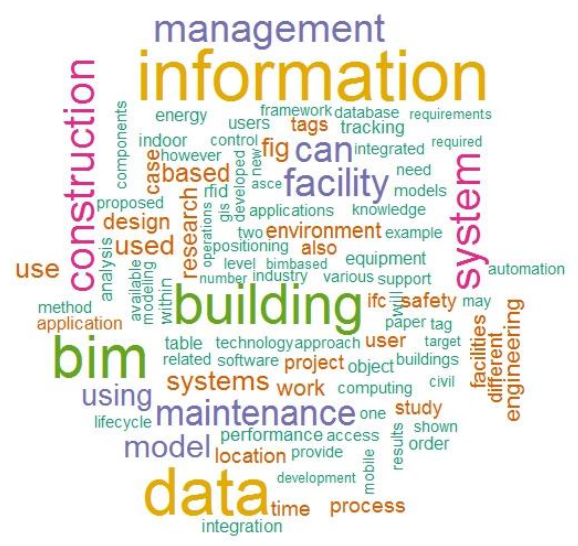

Figure 4: The word cloud of most frequent words.

The final stage of the text mining process was the generation of the cluster dendrogram. A dendrogram is known as a tree type diagram which illustrates how clusters between terms are produced as a result of hierarchical clustering. The hierarchical clustering is a method used in data and text mining to represent the occurrences of entities in form of groups by focusing on generation of hierarchic clusters. The $\mathrm{R}$ package 'cluster' is used to compute the distance matrix between the terms. The matrix is based on the Euclidian distance between clusters. Figure 5 provides a cluster dendrogram generated for this study.

The cluster dendrogram presented in Figure 5 indicates that most frequently observed words "data", "information" and "bim" mainly appear together in the texts. Similarly, words "system" and "model" appear in combination. Other grouping of frequent words includes "building", "construction", "management", "facility" and "maintenance". The result of analysis points out 3 main clusters which can provide guidance in understanding the research focus of the Indoor FM 
technologies. The details on these clusters are discussed in the conclusion section.

\section{CONCLUSIONS}

The paper firstly presented a literature review on the fields concerned with the technologies related to Indoor Facility Management Systems. Later the results of text mining effort, which concentrated on understanding the research trends is presented in the paper. As a result of the clustering exercise provided in the text mining efforts, 3 significant clusters of words were interpreted by the authors. These clusters point out the major concepts in the field which are identified in the literature review. The clusters were;

C1- data, information, bim

C2- system, model

C3-building, construction, management, facility, maintenance

In $\mathrm{C} 1$ cluster-the existence of "data", "information" and "bim" together as frequent words indicate that, the current -research outputs- in the field focus on value of data and information. Mainly what appears important is BIMs, as the semantic information regarding buildings/indoors is well represented in these models. Also it can be interpreted that the use of 3D Indoor Models is also viewed as important field as BIMs provide representations of indoors with $3 \mathrm{D}$ geometries. On the other hand, the role of Geoinformation is underestimated in indoor FM research. For instance, words such as "coordinates" or "geo" or "geoinformation" do not appear in the most frequent word list.

In C2- the existence of words "system" and "model" together is an indication that the research views the role of information systems and information models as key for Indoor Facilities Management. The existence of "bim" in the $\mathrm{C} 1$ cluster is another proof regarding the importance given to the modeling and management of indoor information. But mostly the viewpoint appears as acquiring / utilizing information from BIMs, but not on utilizing georeferenced models and georeferenced representations of indoors which can be found for example in CityGML LOD4. In addition, neither "gml" nor "citygml" were in the frequent word list while the word for the BIM standard "IFC" was there.

In the C3- the third cluster, "Building" and "facility" defines an indoor environment. The papers investigated also indicate the importance of the "construction" phase of the building or facility, as the word "construction" appears in most frequent word list. The words "management" and "maintenance" is related directly with FM knowledge domain, thus the frequent occurrence of these words is natural.

In summary, the most prominent result of the review and the text mining exercise conducted, was the lack of the focus related to the Geoinformation and Geoinformation systems for facilitating the tasks in Indoor Facility Management. In fact, it is foreseeable that FM for complex facilities which span over large areas (such as campus buildings, factories etc.) will require the utilization of geoinformation. Thus, although the role of Geoinformation seems to be underestimated today by researchers of this field, the authors view the integration of GIS - BIM tools, as an apparent need. The next stage of the research the text mining effort will be carried out by focusing on the role of the phrases instead of single words.

\section{REFERENCES}

Akcamete,A., Akinci, B., Garrett, J.H., 2010. Potential utilization of building information models for planning maintenance activities. Proc. Int. Conf. Comput. Civ. Build. Eng. 151-157.

Akula, M., Dong, S., Kamat, V.R., Ojeda, L., Borrell, A., Borenstein, J., 2011. Integration of infrastructure based positioning systems and inertial navigation for ubiquitous context-aware engineering applications. Adv. Eng. Informatics $25,640-655$.

Ammari, K. El, Hammad, A., 2014. Collaborative BIM-Based Markerless Mixed Reality Framework for Facilities Maintenance, in: Computing in Civil and Building Engineering (2014). American Society of Civil Engineers, Reston, VA, pp. 657-664.

Becerik-Gerber, B., Jazizadeh, F., Li, N., Calis, G., 2011. Application Areas and Data Requirements for BIM-Enabled Facilities Management.

Cao, Y., Song, X., Wang, T., 2015. Development of an EnergyAware Intelligent Facility Management System for Campus Facilities. Procedia Eng. 118, 449-456.

Gheisari, M., Goodman, S., Schmidt, J., Williams, G., Irizarry, J., 2014. Exploring BIM and Mobile Augmented Reality Use in Facilities Management, in: Construction Research Congress 2014. American Society of Civil Engineers, Reston, VA, pp. 1941-1950.

Hua, Y., Göçer, Ö., Göçer, K., 2014. Spatial mapping of occupant satisfaction and indoor environment quality in a LEED platinum campus building. Build. Environ. 79, 124-137.

Kang, T.-W., Choi, H.-S., 2015. BIM perspective definition metadata for interworking facility management data. Adv. Eng. Informatics 29, 958-970.

Kang, T.W., Hong, C.H., 2015. A study on software architecture for effective BIM/GIS-based facility management data integration. Autom. Constr.

$54,25-38$

Khaja, M., Seo, J.D., McArthur, J.J., 2016. Optimizing BIM Metadata Manipulation Using Parametric Tools. Procedia Eng. 145, 259-266.

Kim, K., Yu, J., 2016. Improvement of Facility Condition Assessment Processes Using BIM Data, in: Construction Research Congress 2016. American Society of Civil Engineers, Reston, VA, pp. 2432-2442.

Koch, C., Neges, M., König, M., Abramovici, M., 2014. Natural markers for augmented reality-based indoor navigation and facility maintenance. Autom. Constr. 48, 18-30.

Lai, J.H.K., Yik, F.W.H., 2009. Perception of importance and performance of the indoor environmental quality of high-rise residential buildings. Build. Environ. 44, 352-360.

Lee, S., Akin, Ö., 2011. Augmented reality-based computational fieldwork support for equipment operations and maintenance. Autom. Constr. 20, 338-352. 
Lei Song, L., Yongcai Wang, Y., 2011. SpaceAnnotator: a high precision location based asset management system in indoor environment, in: IET International Conference on Communication Technology and Application (ICCTA 2011). IET, pp. 647-651.

Li, H., Chan, G., Wong, J.K.W., Skitmore, M., 2016. Real-time locating systems applications in construction. Autom. Constr. $63,37-47$.

Liu, R., Issa, R.R.A., 2013. Issues in BIM for Facility Management from Industry Practitioners' Perspectives, in: Computing in Civil Engineering. American Society of Civil Engineers, Reston, VA, pp. 411-418.

Liu, R., Issa, R.R.A., 2012. 3D Visualization of Sub-Surface Pipelines in Connection with the Building Utilities: Integrating GIS and BIM for Facility Management, in: Computing in Civil Engineering (2012). American Society of Civil Engineers, Reston, VA, pp. 341-348.

Marzouk, M., Abdelaty, A., 2014. Monitoring thermal comfort in subways using building information modeling. Energy Build. $84,252-257$.

McArthur, J.J., 2015. A Building Information Management (BIM) Framework and Supporting Case Study for Existing Building Operations, Maintenance and Sustainability. Procedia Eng. 118, 1104-1111.

Motamedi, A., Hammad, A., Asen, Y., 2014. Knowledgeassisted BIM-based visual analytics for failure root cause detection in facilities management. Autom. Constr. 43, 73-83.

Motamedi, A., Saini, R., Hammad, A., Zhu, B., 2011. Rolebased access to facilities lifecycle information on RFID tags. Adv. Eng. Informatics 25, 559-568.

Shen, W., Hao, Q., Xue, Y., 2012. A loosely coupled system integration approach for decision support in facility management and maintenance. Autom. Constr. 25, 41-48.

Shi, Y., Du, J., Lavy, S., Zhao, D., 2016. A Multiuser Shared Virtual Environment for Facility Management. Procedia Eng. $145,120-127$.

Skandhakumar, N., Salim, F., Reid, J., Drogemuller, R., Dawson, E., 2016. Graph theory based representation of building information models for access control applications. Autom. Constr. 68, 44-51.

Terreno, S., Anumba, C.J., Gannon, E., Dubler, C., 2015. The Benefits of BIM Integration with Facilities Management: A Preliminary Case Study, in: Computing in Civil Engineering 2015. American Society of Civil Engineers, Reston, VA, pp. 675-683.

Wang, Z., Bulbul, T., Lucas, J., 2015. A Case Study of BIMBased Model Adaptation for Healthcare Facility ManagementInformation Needs Analysis, in: Computing in Civil Engineering 2015. American Society of Civil Engineers, Reston, VA, pp. 395-402.
Wetzel, E.M., Thabet, W.Y., 2015. The use of a BIM-based framework to support safe facility management processes. Autom. Constr. 60, 12-24.

Wu, W., Yang, X., Fan, Q., 2014. GIS-BIM Based Virtual Facility Energy Assessment (VFEA)—Framework Development and Use Case of California State University, Fresno, in: Computing in Civil and Building Engineering (2014). American Society of Civil Engineers, Reston, VA, pp. 339-346.

GitHub Arc12 (2016) Available online: https://github.com/arc12/Text-Mining-Weak-

Signals/wiki/Standard-set-of-english-stopwords

Tableau (2016) Available online at : http://public.tableau.com 


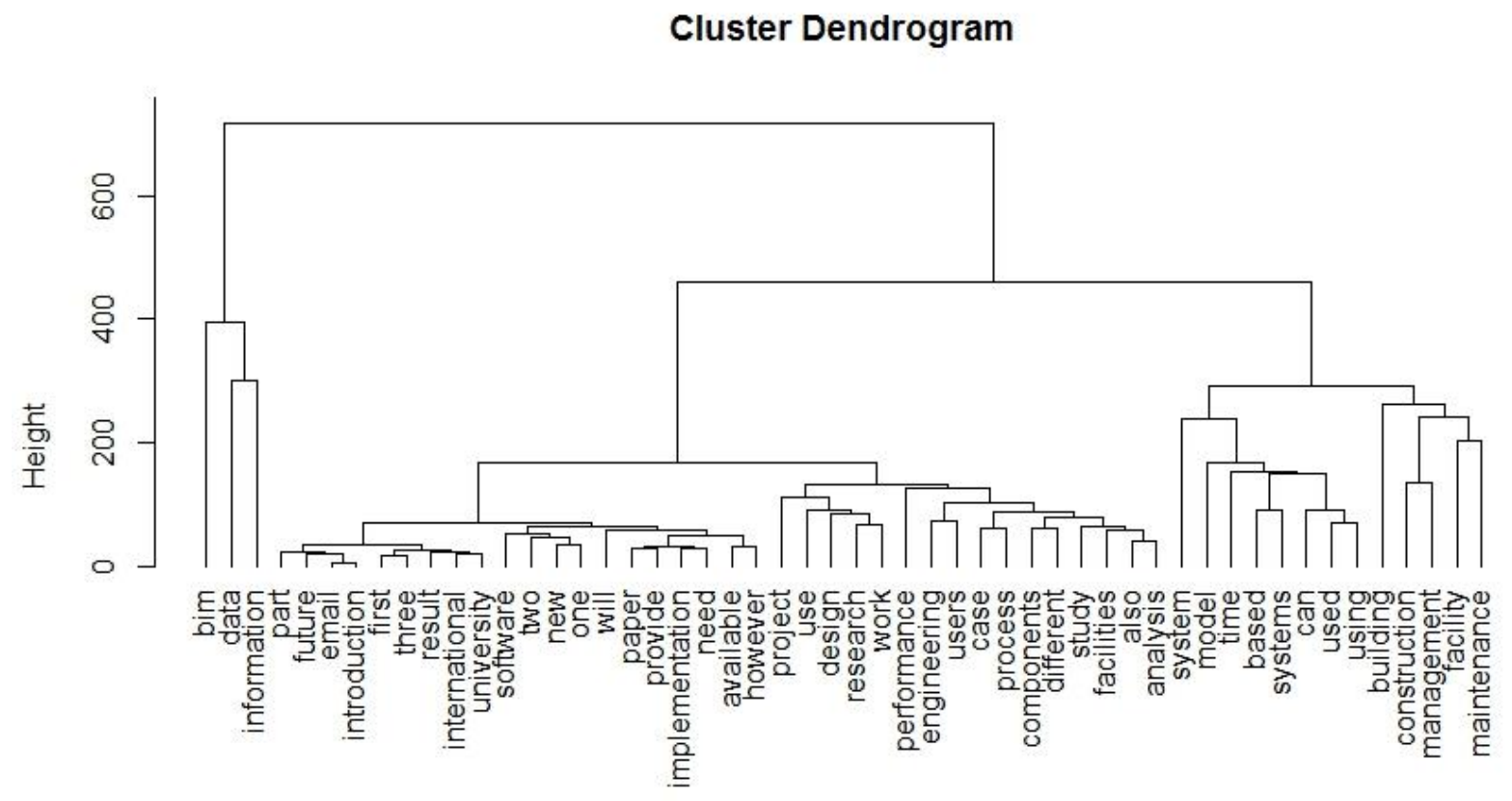

d

Figure 5 The Cluster Dendrogram

hclust (*, "ward.D2") 